\title{
Real-Time Smoke Detection in Video Sequences: Combined Approach
}

\author{
Malenichev Anton and Krasotkina Olga \\ Tula State University \\ Cybernetics faculty \\ Tula, Russia \\ malenichev@mail.ru, O.V.Krasotkina@yandex.ru
}

\begin{abstract}
This paper presents a combined approach for rapid smoke detection from video sequences using pre-improvement methods. Smoke is characterized by different properties like a color, irregularities in motion, smoothing the edges, etc. There are hard to describe it using basic image features. Usually smoke detection algorithms use some color and turbulence features for the smoke description. But our experiments shown the high false alarm rate of such algorithms. In our work we propose the additional smoke description features based on smoke transparency. Before the recognition we use the regions matching method to increase the flexibility of our system. As a first step in processing we extract background. Moving objects are candidates for smoke. The Gray World algorithm is used here. Compare the results with the original frames in order to get image features within some particular gray scale interval. After that we use the rate of color changing to checking of the transparency of current area. Last we calculate complexity of turbulent phenomena of the smoke shape and apply it to the incoming video stream. As a result we have just smoke regions on the video stream. There are different objects, shadows or illumination changes will not be mistaken for smoke by the algorithm. This method gives an early recognition of smoke in the observed scene.
\end{abstract}

\section{Introduction}

In many extreme situations there is visible smoke spread prior to flames. This fact can be of great help for high-efficiency fire control. Using video systems that are too sensitive to smoke can save many lives in dangerous situations. Time is a main factor for minimizing damages caused by fire and smoke. A short distance to the location of fire ignition is a crucial requirement for most of the traditional smoke sensors in order to be efficient. In many cases the fire is already in place when a chemical alarm is going off and the situation is out of control. Video smoke detection has low cost, high efficiency and low maintenance and becomes increasingly important because of it 1] [4. Video is a good option for large, open areas like forest fires, saw mills, petrochemical refineries, etc. Video is a volume sensor as opposed to a point sensor, which looks at just one point in space. Smoke or fire can be not at that point, so the smoke alarm would not be caused. 
A volume video sensor has higher probability of early smoke or flame detection and monitors a larger area [7].

However, video smoke detections have some drawbacks. There are high rate of false alarm, the difficulty of defining smoke in terms of primitive image features such as motion, obscuration, intensity, edge, etc.

There are several smoke detection methods from video captured in visiblespectrum have been proposed. They use of color, motion and geometry of smoke regions. Then apply rule-based analysis or Bayesian analysis to detect the smoke. Out of the many approaches that address these issues some classification could be in place:

1. Using the fractal encoding concepts to find smoke areas from an image. This approach used the feature of smoke to self-similarity of shapes [5].

2. Definition of irregularities in motion due to non-rigidity of smoke. Here computed optical flow field using two neighbor images. Making use of entropy of the distribution of the motion directions It can be possible to separate smoked motion from non-smoked [8].

3. Extracting of image features such as flickering, motion, edge-blurring to segment moving, and edge-blurring regions out from video. The methods to extract these features were background subtraction, temporal wavelet transformation, and spatial wavelet transformation [10].

4. Extracting of local motions from cluster analysis of points in a multidimensional temporal embedding space in order to track local dynamic envelopes of pixels, and then used features of the velocity distribution histogram to discriminate between smoke and various natural phenomena such as clouds and wind-tossed trees that may cause such envelopes [11].

5. Using of different image features, such as the percentage of image change, correlation, variance etc., extracted from both background image and current image, and then used a rule-based or a rule-first-Bayesian-next analysis method to differentiate smoke motion from non-smoke motion [6].

As we can see, there are many approaches that address to these issues, but the main novelty of this work consists in the analysis the smoothing edges of objects beyond the smoke area.

First we make the RGB balancing of the each frame to obtain a real color level regardless of automatic white level adjustment, used in most video cameras. Next we use the color and motion threshold transformations by color and motion to determine regions of interest, which may contain the smoke. Next, most important step - checking the rate of change of color in areas of interest for $\mathrm{N}$ sequential frames. If a change in a particular pixel is more than a predetermined threshold, it is deemed opaque gray object. When the color change does not exceed the predetermined threshold, then we consider it a fact of the presence of smoke in the considered pixel. Next, if the percentage of pixels with smoke in a particular area of interest over a predetermined percentage, the area considered like a smoke. Finally we analyze the behavior of the area of the interest using the turbulence function and with high probability can determine presence or ab- 
sence the smoke in current area. This simple and not time consuming algorithm allows with high accuracy detect smoke.

We aim to present a method that detects smoke in an early stage from video.

\section{Detection of Smoke from Video}

\subsection{Areas Matching}

During recognition the frame can contain multiple objects simultaneously. Therefore it is incorrect to apply the methods of dynamic analysis for all areas of interest as for a single area. We need to operate on local areas, not their totality. To solve this problem we use the areas matching method. Each region of interest in the frame may be regarded as:

1. First time appeared area (without parent area);

2. An area, changed its location and size (has one parent area);

3. An area, resulted as merging some parent areas;

4. An area, resulted as partitioning of the parent area;

The matching algorithm consist of following steps. Each of the $M$ regions on the frame $(i+1)$ subjects operation of "logical and" with each of the $N$ regions in the $i$ frame and then areas of each intersection are determines. The result is stored in the matrix $R(m * n)$. The higher value of element correspond to the closer regions. Zero value means that areas does not intersect. Next we analyze the matrix $\mathrm{R}$ :

1. If all values in a row are zeros, then an area is new (without parent regions);

2. If a row have one non-zero value, then properties are inherited from one parent region;

3. If a row have more than one non-zero value, then the parent region is selected with the maximum area of intersection;

This algorithm has linear complexity, allow us to produce region matching easily and accurately and increase flexibility of our smoke recognition system.

\subsection{Smoke Detection Algorithm}

The first stage of video processing is a balancing of the color scheme of the frames. We calculate the average value of each $R, G, B$ color component to receiving real level of gray color. It averages the RGB of the whole image and then it uses like a balancing constant.

The following stage of processing is a detection of a smoke regions on frames. This procedure is carried out in some steps. For smoke definition used chromatic characteristics of smoke areas. It is known that the smoke has color from light to dark gray. One of the ways to describe color feature of the smoke regions of an RGB image is in 3 :

$$
|R-G|<T ;|G-B|<T ;|R-B|<T ;
$$


where $T$ is a threshold, adjusted on training video set. Naturally, use of a chromatic characteristic for localization of smoke areas isn't enough. Moving of the smoke areas relatively to background frame can also be considered as the property of smoke. Therefore the following stage is detection of moving objects.

Moving areas detection procedure is carried out by recalculating the background image in the sequence of frames. Next the moving objects calculated with subtraction of each color component of the image from background and dissectings away on a threshold. The threshold value is choosed by training sequence of videos. Potentially smoke areas are estimated as combination of moving areas and grey colored areas. Then we analyze the rate of change of color in them.

When we see a scene of smoke, the color of the objects behind it does not change instantly. As soon as the smoke condensed, the color of the object is gradually replaced by the color of the smoke. Let $\Delta R, \Delta G, \Delta B$ - the absolute change in the color of a pixel regions of interest for the $N$ frames. Define the threshold of maximum color change $C_{\max }$. The criterion of the presence of smoke in the area will be fulfillment of conditions:

$$
\Delta R<C_{\max } ; \Delta G<C_{\max } ; \Delta B<C_{\max }
$$

As we are dealing with smoke, its natural phenomena is that it strives to move up. This feature can be detected by monitoring positive local extrema of the turbulence (3).

$$
\Omega=\frac{P(t)}{2 \sqrt{\pi \cdot A(t)}}
$$

where $P(t)$ - perimeter, $A(t)$ - area of region. To estimate of smoke areas perimeter on each frame we use the median filtration procedure after localization of potential smoke areas on them. Rate of change of turbulence in the image is calculated using (4).

$$
\frac{d \Omega(t)}{d t}=\frac{2 P^{\prime}(t) A(t)-P(t) A^{\prime}(t)}{4 \sqrt{\pi \cdot A^{3}(t)}}
$$

where $P$ represents the perimeter/edges of the remaining moving gray features and $A$ represents the area of the region. As the complexity of a shape increases (i.e., the perimeter increase with respect to the area) the value associated with $\frac{d \Omega(t)}{d t}$ increases.

As a last step we investigate the cumulative sum of each area for each frame and the obtained $\frac{d \Omega(t)}{d t}$ in this frame and create a set of value intervals, which are valid for smoke.

\section{Experimental Research}

Experimental study is to test the developed system on a series of 20 videos, half of which contain smoke (1-10 rows in the table 1), another half - not (11-20 rows in the table 1). Testing was performed using color features only, motion 
features only and two types together. In all cases we, first, allocate a region of potential smoke using color or moving features and divide it to separate areas. Then we apply turbulence function and transparency analysis on them to smoke recognition. Testing was conducted at the same optimal settings of the system and for each video sequence is determined errors of the first (false reject rate FRR) and second kind (false accept rate - FAR).

Table 1. Results of experiments with optimal settings

\begin{tabular}{|c|c|cc|cc|cc|}
\hline \multirow{2}{*}{ № } & FileName & \multicolumn{2}{|c|}{ Color + turbulence } & \multicolumn{2}{|l|}{ Moving + turbulence } & \multicolumn{2}{|c|}{ All features } \\
& FRR & FAR & FRR & FAR & FRR FAR \\
\hline 1 & Clip_23_Smoke & 0,13 & 0,17 & 0,32 & 0,12 & 0 & 0 \\
2 & ModifiedClip_31 & 0,12 & 0,08 & 0,2 & 0,07 & 0.3 & 0 \\
3 & sBehindtheFence & 0,22 & 0,42 & 0,09 & 0,22 & 0,03 & 0,05 \\
4 & sBtFence2 & 0,1 & 0,33 & 0,09 & 0,17 & 0 & 0,02 \\
5 & sEmptyR1 & 0,22 & 0,5 & 0,17 & 0,42 & 0,01 & 0,05 \\
6 & sEmptyR2 & 0,47 & 0,42 & 0,43 & 0,39 & 0,22 & 0,27 \\
7 & ShorterIsyamNight_Short & 0,29 & 0,55 & 0,25 & 0,35 & 0,15 & 0,02 \\
8 & Smoke_Manavgat_Raw & 0,01 & 0,70 & 0,01 & 0,03 & 0,01 & 0 \\
9 & sParkingLot & 0,26 & 0,55 & 0,21 & 0,27 & 0,11 & 0,02 \\
10 & sWasteBasket & 0,07 & 0,28 & 0,05 & 0,07 & 0.01 & 0 \\
\hline 11 & Astak IP Cam & 0 & 0,07 & 0 & 0,05 & 0 & 0 \\
12 & AXIS 212 PTZ & 0 & 0,11 & 0 & 0,08 & 0 & 0 \\
13 & CCTV & 0 & 0,17 & 0 & 0,13 & 0 & 0,02 \\
14 & Motion Test & 0 & 0,22 & 0 & 0,17 & 0 & 0 \\
15 & my IPcamera 360 & 0 & 0,33 & 0 & 0,26 & 0 & 0 \\
16 & RVi-127 & 0 & 0,3 & 0 & 0,21 & 0 & 0 \\
17 & surveillance Camera & 0 & 0,22 & 0 & 0,13 & 0 & 0 \\
18 & Ubiquiti airCam & 0 & 0,18 & 0 & 0,14 & 0 & 0 \\
19 & VeSta VC-6320 IR & 0 & 0,19 & 0 & 0,17 & 0 & 0 \\
20 & Vivotek IP8332 & 0 & 0,13 & 0 & 0,14 & 0 & 0 \\
\hline
\end{tabular}

For video set from table 1 average values of FAR and FRR are 0.04 and 0.02 respectively. The maximum error value is 0.27 . This is due to the rather complicated scenes and specificity of smoke in the "complex" video sequences. The especial interesting video is the video sequence ShorterIsyamNight, which in addition to the smoke contain the object - a man in a black and gray clothes, appered in a front of camera and walked down the hall, away from the viewpoint. The background color is mostly gray and adds additional complexity for recognition. As can be seen from the figure, a third-party object (in this case a man) is not recognized by the system as smoke. Smoke on the contrary, well-allocated by the system.

All video sequences are publicly available at the URL: http://yadi.sk/d/q97BQ9v58WNRD. 


\section{Conclusion}

In this paper we have shown an approach for early smoke detection. The main difference between smoke detection algorithms presented earlier is that in this work we analysis the speed of color changing of objects beyond the smoke area. This allows to detect smoke regions most robustly. Average false reject and false accept rate is about $5 \%$. Disadvantage of this system - manual setting. The matter of future work is developing of autotune system.

\section{References}

1. Buchsbaum, G.: A spatial processor model for object color perception. J. Franklin Inst. 310(1), 1-26 (1980)

2. Catrakis, H.J., Dimotakis, P.E.: Shape Complexity in Turbulence. J. Phys. Rev. Lett. 80(5), 968-971 (1998)

3. Celik, T., Ozkaramanly, H., Demire, H.: Fire and Smoke Detection Without Sensors: Image Processing Approach. In: Proceedings of 15th European Signal Processing Conference EUSIPCO, pp. 1794-1798 (2007)

4. Chunyu, Y., Jun, F., Jinjun, W., Yongming, Z.: Video Fire Smoke Detection Using Motion and Color Features. J. Fire Technology 46(3), 651-663 (2010)

5. Fujiwara, N., Terada, K.: Extraction of a smoke region using fractal coding. In: IEEE International Symposium on Communications and Information Technology, ISCIT 2004, October 26-29, vol. 2, pp. 659-662 (2004)

6. Grech-Cini, H.J.: Smoke Detection. US Patent No. US6844818B2

7. Gudukbayb, U., Enis Cetin, A.: Computer vision based method for real-time fire and flame detection. Pattern Recognition Letters 27(1), 49-58 (2006)

8. Kopilovic, I., Vagvolgyi, B., Sziranyi, T.: Application of panoramic annular lens for motion analysis tasks: surveillance and smoke detection. In: Proceedings of 15th International Conference on Pattern Recognition, September 3-7, vol. 4, pp. 714-717 (2000)

9. Toreyin, B.U., Dedeoglu, Y., Cetin, A.E.: Contour Based Smoke Detection in Video Using Wavelets. In: Proceedings European Signal Processing Conference, EUSIPCO 2006 (2006)

10. Toreyin, B.U., Dedeoglu, Y., Cetin, A.E.: Wavelet based real-time smoke detection in video. In: Procedings of EUSIPCO 2005 (2005)

11. Vicente, J., Guillemant, P.: An image processing technique for automatically detecting forest fire. International Journal of Thermal Sciences 41(12), 1113-1120 (2002) 